\title{
Estudio Electroquímico del Ácido 3,4- Dihidroxifenilacético (DOPAC) Adsorbido sobre Oro
}

Sylvia M. Esquenoni, Alicia E. Von Mengershausen y María G. Sustersic

Universidad Nacional de San Luis, Facultad de Ingeniería y Ciencias Económico-Sociales, 25 de Mayo 384.5730 Villa Mercedes, San Luis-Argentina.(e-mail: masus@fices.unsl.edu.ar)

\section{Resumen}

El objetivo de este estudio es conocer el comportamiento del ácido 3,4 dihidroxifenilacético (DOPAC) como adsorbato y como reactivo electroquímico adsorbido sobre oro. Las características de la reacción redox del anillo catecol permite estudiar esta sustancia adsorbida a circuito abierto. El procedimiento consiste en las etapas de adsorción, enjuague con el electrolito y barridos de voltametría cíclica para determinar la cantidad de sustancia adsorbida y el comportamiento electroquímico de la misma. Se trabajó con dos celdas, una de adsorción y otra electroquímica, termostatizadas a $25^{\circ} \mathrm{C}$. La adsorción del DOPAC es débil, consolidándose el enlace con el tiempo para formar una ligadura tipo $\sigma$. Se determina un área por molécula de $47 \AA^{2}$, similar a la obtenida para la dopamina.

Palabras clave: adsorción DOPAC, voltametría, oro, estudio electroquímico

\section{Electrochemical Study of 3,4-Dihydroxyphenylacetic Acid (DOPAC) Adsorbed on Gold}

\begin{abstract}
The objective of this study was to obtain knowledge on the behavior of acid 3,4 dihydroxyphenylacetic (DOPAC) as an adsorbate and electrochemical reagent adsorbed on gold. The redox reaction characteristic of the catechol ring allows the study of the substance adsorption under open circuit conditions. The step of the procedure include adsorption, rinsing with electrolyte, and making cyclic voltammetric scan to determine the amount of adsorbed substance and its electrochemical behavior. For this, two cells were employed, one for adsorption, and the other electrochemical, both were thermostatted at $25^{\circ} \mathrm{C}$. The DOPAC adsorption is weak. The adsorption bound is strengthening with the time forming a $\sigma$ bound. The area per molecule of $47 \AA^{2}$, very near of that of the dopamine is obtained.
\end{abstract}

Keywords: DOPAC adsorption, voltammetry, gold, electrochemical study 


\section{INTRODUCCION}

El ácido di-hidroxi-fenil-acético (DOPAC) es el metabolito más importante de la dopamina. Se produce por acción de la mono-amino-oxidasa (MAO) sobre la dopamina en las terminales nerviosas de las células dopaminérgicas. La catecol-o-metil-transferasa degrada la dopamina para dar 3-metoxi-tiramina la cual, por acción de la MAO, produce ácido homovanílico. Ambos metabolitos se determinan en la orina y cuantifican la cantidad total de dopamina metabolizada. Se hace una separación de los distintos metabolitos por cromatografía líquida y se determina el DOPAC mediante técnicas voltamperométricas (Eisenhover et al., 1986).

Por otra parte, los investigadores en neurociencia requieren el desarrollo de técnicas electroanalíticas para determinar neurotransmisores y sus metabolitos en el fluido extracelular del cerebro. Hasta ahora, solo se practicó el análisis en cerebros de animales con electrodos específicos (Gonon et al., 1981; Ponchon et al., 1979) y técnicas voltamétricas especiales (Shenk et al., 1983; Adams,1976; Lane et al., 1976). Por tratarse de determinaciones electroanalíticas, resulta de interés conocer el comportamiento electroquímico de la sustancia, así también como su comportamiento como adsorbato, ya que la adsorción es una etapa de la reacción redox global.

Las sustancias orgánicas que presentan anillos aromáticos con grupos $\mathrm{OH}$ en posiciones orto y para, se oxidan de tres maneras distintas: una, a potenciales bajos, donde se oxidan reversiblemente los grupos $\mathrm{OH}$ del anillo, otra, a potenciales intermedios, donde se adiciona oxígeno al anillo y una tercera, a potenciales altos, donde se rompen los enlaces carbonocarbono. Se cree que el segundo tipo de reacción ocurre por mediación del óxido incipiente hidratado, pues ocurre en los potenciales en los cuales este óxido se forma (Burke, 1994). En cambio, el tercer tipo de oxidación ocurre simultáneamente con la formación del óxido de oro (Sustersic et al., 1996) y a potenciales superiores. Aquí, se estudia el primer tipo de reacción y, además, se la aplica para determinar concentraciones superficiales de sustancia adsorbida.

\section{PARTE EXPERIMENTAL}

Se trabajó con dos celdas, una de adsorción y otra electroquímica, termostatizadas a $25^{\circ} \mathrm{C}$. En ambas se introducía una solución de ácido sulfúrico $0,5 \mathrm{M}$. El reactivo se disolvía en la solución contenida en la celda de adsorción. El electrodo de trabajo consistía en un alambre de oro de $3 \mathrm{~cm}$ de longitud y $1 \mathrm{~mm}$ de diámetro. Su área real se obtenía midiendo la carga de formación electroquímica de una monocapa de óxido de oro (Michri et al., 1972) Una chapa de oro de $4 \mathrm{~cm}^{2}$ de superficie, se utilizó como contraelectrodo.

La celda electroquímica era de vidrio pyrex de dos compartimentos. Los potenciales se midieron con respecto al electrodo reversible de hidrógeno instalado en un compartimento separado y conectado con el compartimento central mediante un capilar de Luggin.

El electrodo se trataba en la celda electroquímica con barridos entre $0,1 \mathrm{~V}$ y $1,7 \mathrm{~V}$ a $0,04 \mathrm{~V} / \mathrm{seg}$ hasta obtener un registro reproducible. Luego se llevaba a la celda de adsorción durante un tiempo t, se extraía, se enjuagaba con la solución de electrolito y se llevaba nuevamente a la celda electroquímica donde se realizaban barridos de voltametría cíclica destinados a estudiar la capa del reactivo adsorbido.

En algunos casos se utilizó una tercera celda llamada celda de desorción, pues en ella se realizaba la experiencia de desorción física con la cual se verificaba si el reactivo se adsorbía física o químicamente. La solución de electrolito contenida en esta celda era la misma que la contenida en la celda electroquímica $y$, en la misma forma, se termostatizaba y saturaba con nitrógeno. El burbujeo de este gas mantenía al líquido libre de oxígeno atmosférico y producía turbulencias que favorecían la separación de la sustancia débilmente ligada (Almeida et al., 2000)

\section{RESULTADOS Y DISCUSION}

\section{Comportamiento de la capa adsorbida}

El perfil potencial-corriente muestra un pico de corriente anódica en $0,8 \mathrm{~V}$ y uno complementario, de corriente catódica en $0,77 \mathrm{~V}$. con dos hombros en 0,9 y $1,0 \mathrm{~V}$ (Figura 1). En el barrido inverso, aparece un pico de corriente catódica a $0,67 \mathrm{~V}$ y dos ondas de corriente catódica en $0,55 \vee$ y $0,57 \mathrm{~V}$ respectivamente. En los ciclos sucesivos, desaparecen las contribuciones en los potenciales más positivo y más negativo.

Información Tecnológica - Vol. 18 No 1 - 2007 
La cupla principal de picos de corriente, simétrica con respecto a $0,785 \mathrm{~V}$, se comporta como el conjunto de dos picos originados por la oxidación reversible de los grupos $\mathrm{OH}$ sobre el anillo catecol. El potencial de esta cupla es ligeramente menor que el de la misma reacción en las catecolaminas (Sustersic et al., 1996) Esto se debe a que el efecto de desactivación de la cadena lateral sobre la reacción electrofílica en el anillo es menor por la presencia del grupo carboxilo.

La reacción que ocurre a potenciales más altos se atribuye a la decarboxilación.

\section{Comportamiento del adsorbato}

Después del primer ciclo, parte de la sustancia permanece adsorbida y parte se desorbe como se puede ver en el segundo ciclo del voltamperograma (Figura 1). Comparando el tercer ciclo con el segundo y así sucesivamente, se puede hacer la misma observación.

Además, si el electrodo, con la capa de reactivo adsorbido se deja durante 30' en la celda de desorción, se elimina casi toda la sustancia presente sobre la superficie (Figura 2a).

Esto no sucede cuando los tiempos de adsorción son largos (180 min). En este caso, se elimina la sustancia que reacciona a los potenciales más bajos permaneciendo aquella que reacciona a potenciales mayores (Figura $2 b)$.

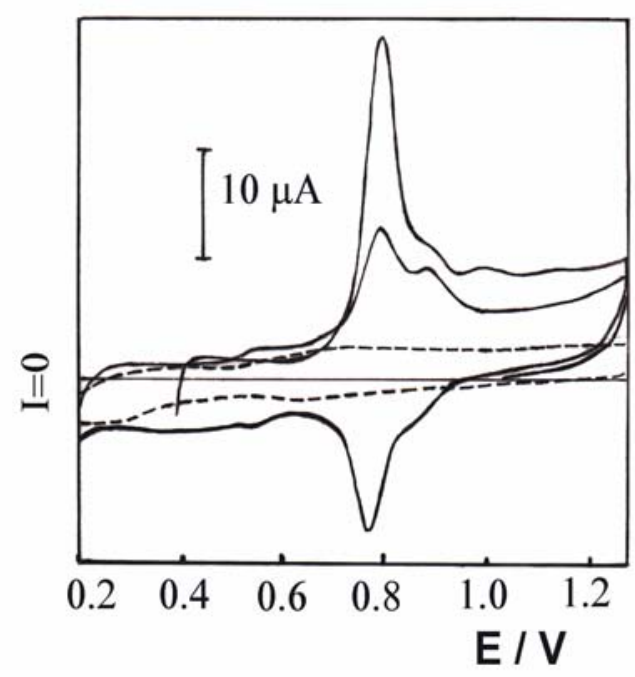

Fig.1: Voltamperograma de la electrooxidacion de dopac adsorbido sobre oro. $\mathrm{T}=25^{\circ} \mathrm{C}$, $\mathrm{t}=5 \mathrm{~min}, \mathrm{c}=5.10^{-2} \mathrm{M}, \mathrm{v}=10 \mathrm{mV} \cdot \mathrm{s}^{-1}$

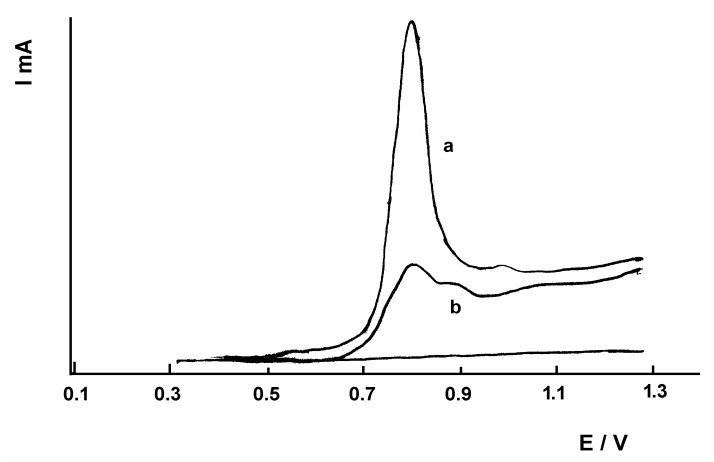

Fig2a: Voltamperograma obtenido de un electrodo recubierto de dopac. a) $\mathrm{c}=2,5 \cdot 10^{-2} \mathrm{M}$. b) $\mathrm{c}=2,5.10^{-2} \mathrm{M}$ introduciendo el paso de desorción de 30 minutos. $\mathrm{T}=25^{\circ} \mathrm{C}, \mathrm{t}=5 \mathrm{~min}$, $\mathrm{v}=10 \mathrm{mV} \cdot \mathrm{s}^{-1}$

Se evaluaron las cargas de reacción anódica y el área por molécula y se graficaron en función de c y t (Figuras 3 a 6 ). En la evaluación se ha tenido en cuenta la transferencia de dos electrones por molécula. Como la sustancia posee dos grupos de reacción, habría que considerar los electrones puestos en juego en ambas reacciones. Sin embargo, es poco probable que la misma molécula participe en ambas reacciones en el mismo barrido potenciodinámico.

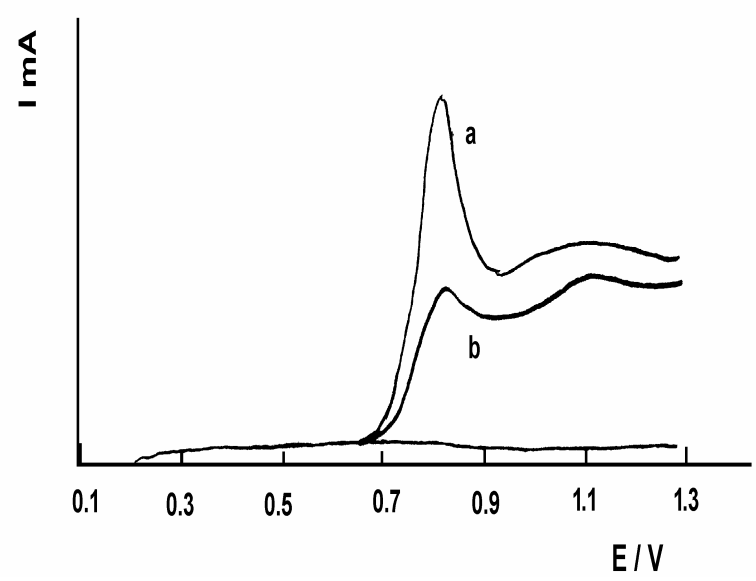

Fig2b: Voltamperograma obtenido de un electrodo recubierto de dopac. a) $\mathrm{c}=2,5 \cdot 10^{-2} \mathrm{M}$. b) $\mathrm{c}=2,5.10^{-2} \mathrm{M}$ introduciendo el paso de desorción de 30 minutos. $\mathrm{T}=25^{\circ} \mathrm{C}, \mathrm{t}=180$ $\min , \mathrm{v}=10 \mathrm{mV} . \mathrm{s}^{-1}$

Para participar en una reacción, debe tener una orientación y una configuración adecuada. Es poco probable que la misma molécula posea la orientación y la configuración adecuada para participar en ambas reacciones al mismo tiempo. Es más probable, que parte de las moléculas participen en una 
reacción y la otra parte participe en la otra reacción. Es más probable que las áreas por molécula obtenidas bajo esta suposición sean las correctas (Figuras 5 y 6 ).

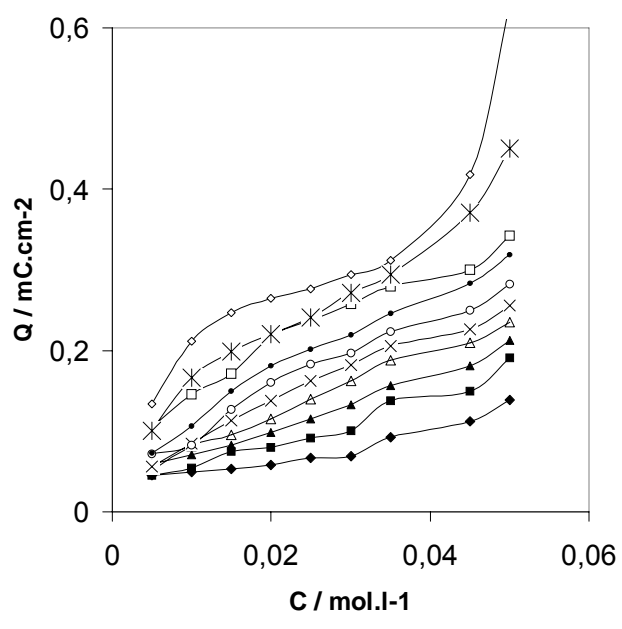

Fig.3: Gráfico de la carga anodica vs concentración. Paramétrica en los siguientes

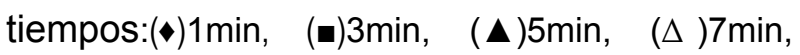
(x)10min, $(\bigcirc) 15 \mathrm{~min},(\bullet) 20 \mathrm{~min},(\square) 30 \mathrm{~min},\left(^{*}\right) 40 \mathrm{~min}$, $(\diamond) 60 \mathrm{~min}$.

La Figura 3 muestra las relaciones carga / concentración para distintos tiempos de adsorción. La curva correspondiente a $60 \mathrm{mi}-$ nutos se podría considerar una isoterma de adsorción. La dependencia de la cantidad adsorbida con el tiempo puede observarse en la Figura 4. Esta figura muestra que, a partir de 30 minutos, la carga varia muy poco con el tiempo. Sin embargo, cambia la distribución de las cargas debajo de los dos picos de corriente anódica (Figura 2b), lo cual significa que cambia la configuración de la sustancia sobre la superficie. Además, a medida que crece el tiempo de adsorción, es mayor la cantidad de sustancia que no se puede eliminar por métodos físicos, lo cual significa que hay una ligadura que se consolida.

Considerando la curva superior en la Figura 3 como isoterma de adsorción, esto es, como una gráfica de equilibrio, ella tiene el aspecto de una correspondiente a la formación de multicapas.

Sin embargo, antes de decidir si se trata de una monocapa o de una multicapa, es necesario observar las curvas del área por molécula en función del tiempo y de la concentración.

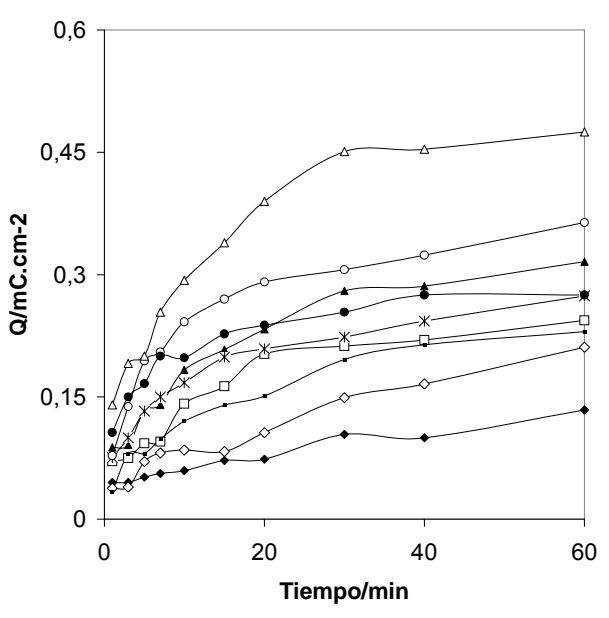

Fig. 4: Gráfico de la carga anodica vs tiempo de adsorción. Parametrica en las siguientes concentraciones: $(\diamond) 5.10^{-3} \mathrm{M},(\diamond) 1.10^{-2} \mathrm{M}$, $(\square) 1,5.10^{-2} \mathrm{M},(\mathbf{\bullet}) 2.10^{-2} \mathrm{M},(\boldsymbol{\Delta}) 2,5.10^{-2} \mathrm{M},\left(^{*}\right) 3.10$ ${ }^{-2} \mathrm{M},(\bigcirc) 3,5.10^{-2} \mathrm{M},(\bullet) 4,5.10^{-2} \mathrm{M},(\Delta) 5.10^{-2} \mathrm{M}$

En la Figura 5, donde se representa el área por molécula en función del tiempo de adsorción para distintas concentraciones, se observa que las curvas correspondientes a las dos concentraciones más diluidas presentan mesetas entre 40 y $50 \AA^{2}$. En la representación del área por molécula en función de la concentración (Figura 6), también se encuentran mesetas entre 40 y $50 \AA^{2}$ para los dos tiempos más cortos. Para el área límite por molécula de la dopamina se encontró un valor aproximado de $49 \AA^{2}$ (Sustersic et al., 1999) de modo que los valores encontrados estarían muy próximos al valor de la dopamina. Esto es razonable, dado que ambas moléculas tienen un tamaño similar.

Cabe ahora decidir si el cambio encontrado en la supuesta isoterma de adsorción se debe a la formación de multicapas ó a un cambio de configuración superficial.

Como para tiempos cortos aparecen mesetas en la gráfica de área por molécula en función del tiempo para las soluciones más diluídas, para estos tiempos cortos ocurre ya un cambio de posición de la molécula sobre la superficie.

Para las soluciones más concentradas, el cambio también ocurre para tiempos cortos, pero los mismos no aparecen como mesetas en las representaciones, por ser muy breves

Información Tecnológica - Vol. 18 No 1 - 2007 
los períodos de cambio. En trabajos previos se vio que este tipo de compuestos se ligan a la superficie mediante el anillo bencénico (Wightman et al., 1988).

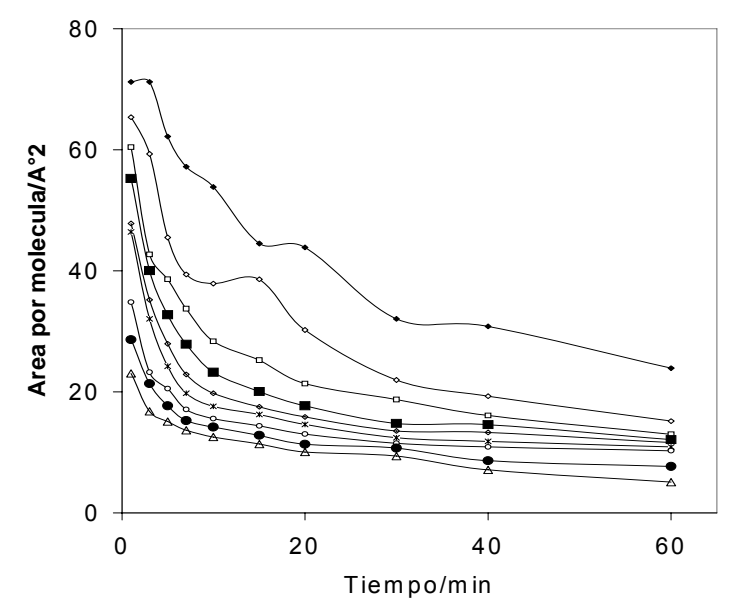

Fig. 5 : Gráfico del área por molécula vs tiempo de adsorción. Parametrica en las siguientes concentraciones: $(\diamond) 5.10^{-3} \mathrm{M},(\diamond) 1.10^{-2} \mathrm{M}$, $(\square) 1,5.10^{-2} \mathrm{M},(\mathbf{\bullet}) 2.10^{-2} \mathrm{M},(\boldsymbol{\Delta}) 2,5.10^{-2} \mathrm{M},\left(^{*}\right) 3.10$ ${ }^{-2} \mathrm{M},(\bigcirc)$ ) $3,5.10^{-2} \mathrm{M},(\bullet)$ 4,5.10 ${ }^{-2} \mathrm{M},(\Delta)$ ) $5.10^{-2} \mathrm{M}$.

El anillo bencénico se adsorbe en dos maneras distintas (Clark, 1970): en forma paralela a la superficie mediante uniones $\pi$, que ligan débilmente a la molécula con la superficie y en forma perpendicular a la superficie con uniones sigma, que ligan fuertemente a la molécula con la superficie.

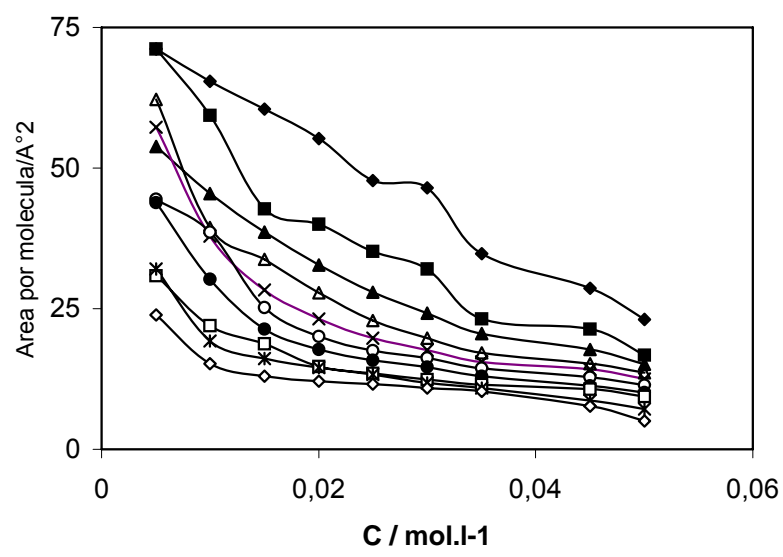

Fig. 6: Gráfico del área por molécula vs concentración. Paramétrica en los siguientes tiempos: $(\bullet) 1 \mathrm{~min}, \quad(\boldsymbol{\bullet}) 3 \mathrm{~min}, \quad(\mathbf{\Delta}) 5 \mathrm{~min}, \quad(\Delta) 7 \mathrm{~min}$, (x)10min, $(\bigcirc) 15 \mathrm{~min},(\bullet) 20 \mathrm{~min},(\square) 30 \mathrm{~min},\left(^{*}\right) 40 \mathrm{~min}$, $(\diamond) 60 \mathrm{~min}$.
La concentración de DOPAC fuertemente adsorbido aumenta con el tiempo de adsorción (Fig $2 b$ ) obteniéndose valores muy pequeños de áreas por molécula. Como siempre hay una cantidad de adsorbato removible por métodos físicos (Fig 2b), no puede descartarse la formación de capas yuxtapuestas y unidas entre sí por enlaces de Van der Waals. Esto se manifiesta particularmente para soluciones concentradas (Fig 3).

Para las soluciones más diluidas, se obtiene una relación lineal entre la carga y la raíz cuadrada del tiempo (Fig 7), señalando un control difusional para la formación de una capa. Esto no ocurre para las soluciones más concentradas, probablemente por causa de la formación de una multicapa. La primera porción de la isoterma de adsorción (Fig 3), se ajusta bastante bien a la ley de Temkin, señalando heterogeneidad en la distribución de la energía de adsorción .

\section{CONCLUSIONES}

EI DOPAC se adsorbe sobre el oro en forma débil, mediante uniones $\pi$ entre el anillo y la superficie. Luego, cambia la configuración paralela de la molécula adsorbida a una perpendicular a la superficie mediante uniones $\sigma$. La primer parte se adsorbe débilmente, mientras que la segunda ligadura es fuerte. Es probable la formación de capas yuxtapuestas unidas entre sí por uniones de Van der Vaals. Estas capas pueden ser removidas por métodos físicos. Esto ocurre para las soluciones más concentradas y para tiempos largos de adsorción. La adsorción está controlada por difusión.

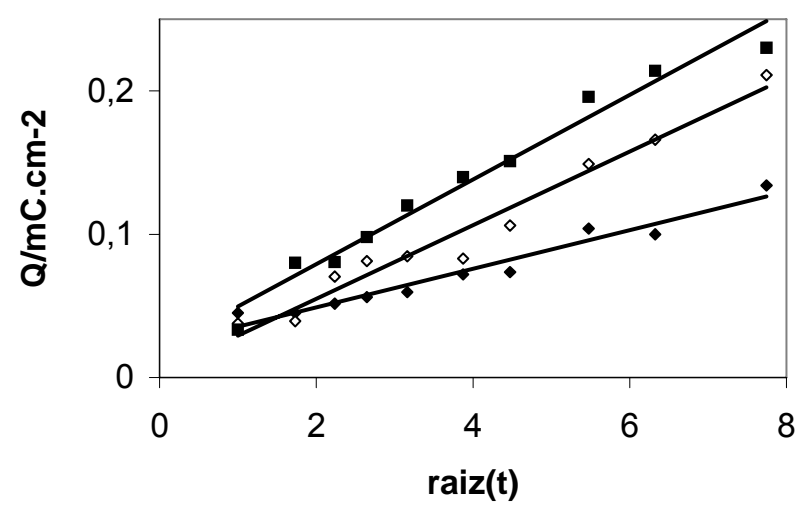

Fig. 7: Gráfico de la carga vs $\sqrt{t}$ para solu-ciones diluidas de DOPAC. ( $) 5.10^{-3}$ $\mathrm{M},(\diamond) 1.10^{-2} \mathrm{M}$ y (๘) $2.10^{-2} \mathrm{M}$. 


\section{REFERENCIAS}

Adams, R.N., Probing Brain Chemistry with Electroanalytical Techniques, Anal. Chem.48, 1128-1137 (1976).

Almeida, N. V. Esquenoni S. M., von Mengershausen A. E., Sustersic, M.G., Estudio electroquímico de la adsorción de hidroquinona sobre oro. Inf. Tecn. 11(5) 71-77 (2000).

Burke, L. D., Premonolayer oxidation and its role in electrocatalysis. Electrochim. Acta 39 (11/12), 1841-1848 (1994).

Clark, A., The Theory of Adsorption and Catalysis. Academic Press. New York (1970).

Eisenhofer,G. y otros 6 autores, Simultaneous liquid-chromatografic determination of 3,4dihydroxyphenilglycol catecholamines and 3,4-dihydroxyphenylalanine in plasma, and their responses to inhibition of monoamine oxidase. Clin.Chem.,32(11)2030-2033 (1986).

Gerhardt, G.A. y otros 6 autores, Nafioncoated electrodes with high selectivity for CNS electrochemistry. Brain Research, 290, 390395 (1984).

Gonon, F. G., Fombarlet, C. M., Buda, M. J. y Pujol, J. F., Electrochemical Treatment of Pyrolytic Carbon Fiber Electrodes. Anal. Chem., 53, 1386-1389 (1981).

Lane, R.N. y Hubbard, A., Differential Double Pulse Voltammetry at Chemically Modified Platinum Electrodes for in vivo. Determination of Catecholamines. Analytical Chemistry, 48 $\mathrm{N}^{\circ} 9,1287$ (1976).

Lane, R.N., y otros 3 autores, Brain catecholamines: Detection in vivo by means of differen- tial pulse voltammetry at surface-modified platinum electrodes. Brain Reseach 114, 346 (1976)

Michri, A., Pshchenichnikov,A.G. y Burshtein, R., Determining the actual surface area of smooth gold electrodes. Elektrokhimiya, 8, 351-352 (1972)

Nagy, G. y otros 7 autores, lon exchange and transport of neurotransmitters in nafion films on conventional and microelectrode surfaces. J. Electroanal. Chem., 188, 85-94 (1985).

Ponchon, J.N. y Cespuglio, R., Normal Pulse Polarography with Carbon Fiber Electrodes for in Vitro and in Vivo Determination of Catecholamines. Anal. Chem., 48, 1483 (1979).

Schenk, J.O., Miller, E.,Adams, R.N., Electrochemical Techniques for The Study of Brian Chemistry. Bioelectrochem., 60, 311 (1983).

Sreenivas, G. y otros 6 autores, Fabrication and Characterization of Sputtered-Carbon microelectrode Arrays. Anal. Chem., 68, 18581864 (1996).

Sustersic M. G., Esquenoni S. M., Zaniolo S. M., Abaca C. R. y Almeida N. V., Comportamiento electroquimico de neurotransmisores adsorbidos sobre oro. An. Asoc. Quim. Argent., 84, 6, 563-572 (1996).

Sustersic, M.G., von Mergershausen, A.E., Esquenoni, S.M., Grzona, C.B., Determinación electroquimica de co-areas de moléculas adsorbidas. Inf. Tecn. 10 (5) 83-89 (1999).

Wightman, R.M., May, L.J., Michael, A.C., Detection of Dopamine Dynamics in the Brain. Anal. Chem.: 60, 769-779 (1988). 